\title{
THE EFFECTIVENESS OF SAFETY CAMPAIGN VMS MESSAGES - A DRIVING SIMULATOR INVESTIGATION
}

\author{
A. Hamish Jamson and Natasha Merat, \\ Institute for Transport Studies, \\ University of Leeds, U.K. \\ E-mail: a.h.jamson@its.leeds.ac.uk
}

\begin{abstract}
Summary: This report outlines an off-road trial of the effectiveness of Safety Campaign Messages (such as "Watch Your Speed") sometimes displayed on Variable Message Signs (VMS) throughout the U.K.'s motorway network. Eighty drivers took part in the driving simulator study. It primarily investigated the effectiveness of the individual messages and how their presence affected driver behaviour towards more critical Tactical Incident Messages (TIM), such as those that might warn of an impending hazard. Both the content and concentration of the Safety Campaign Messages were varied. The Safety Campaign Messages in themselves were not especially beneficial, in that drivers did not significantly modify their driving style purely on the advice of the messages. However, witnessing the odd VMS carrying such a message appeared to improve driver alertness to the context of the VMS and consequently response to a TIM became more timely under these conditions. Yet, if the frequency of Safety Campaign Messages was overly high, drivers became jaded with the VMS content and their ability to act appropriately to a TIM degraded.
\end{abstract}

\section{INTRODUCTION}

In the U.K., responsibility for the motorways and main trunk roads falls to the British Government's Highways Agency (HA). HA have control over the network's Variable Message Signs (VMS) to supply drivers with up-to-date information regarding road and travel conditions. A common use of VMS is to display a Tactical Incident Message (TIM), a specific message warning of a particular impending hazard. HA also use VMS to give more general advice on good driving habits by utilising Safety Campaign Messages. VMS active with a Safety Campaign Message may include such pearls of wisdom as "Watch Your Speed" (WYS) or "Keep Your Distance" (KYD).

There is evidence that VMS warning signs can reduce mean speed (e.g., Rama and Kulmala, 2000). However, overuse of VMS can lead to a reduction of their effectiveness over time (Garber and Patel, 1995). This led HA to commission three on-road investigations to determine the immediate and long-term effects of displaying Safety Campaign Messages on VMS. The three on-road studies undertaken in October 2004, June/July 2005 and January 2006 demonstrated limited effects of some Safety Campaign VMS, but results were far from conclusive.

In the October 2004 trial (Highways Agency, 2005a) for one week, two VMS on either carriageway of the M1 in Derbyshire carried the Safety Campaign Message "Keep Left Unless Overtaking." Over the next few days a very small increase (around 1\%) in the traffic count towards the left-most lane was observed (in direct contrast to the U.S. and most of mainland 
Europe, vehicles in the U.K. drive on the left). A more colloquial version communicating the same meaning, "Don't Hog The Middle Lane," was slightly more successful, with around 2.5\% more traffic observed in the left-most lane. Neither message demonstrated any long-term effects, in that within a week from the start of the trial, traffic counts in each lane returned to those observed before the messages were displayed.

A few months later, in the summer of 2005, an identical presentation of "Keep Left Unless Overtaking" demonstrated no significant effect in shifting traffic towards lane 1 (Highways Agency, 2005b). However, a new message, "Keep Your Distance," did show a small increase (around 5\%) in headway. Another new message, "Watch Your Speed," showed a significant speed reduction of some 3-4\% throughout the trial. Similar results for these two specific messages were also shown during the final trial (Highways Agency, 2006).

The three on-road trials naturally took a macroscopic view of the effectiveness of the Safety Campaign Messages by investigating their role over all the vehicles that pass VMS carrying them. This off-road (driving simulator) study differed in that it was designed to take a more microscopic look at individual driver behaviour under the influence of Safety Campaign VMS. As such, it had three specific aims:

- To determine the immediate and long-term effects of displaying Safety Campaign Messages on VMS.

- To establish how the risk of a driver failing to read a Tactical Incident Message changes as the frequency and concentration of Safety Campaign messages varies.

- To ascertain the optimum level of usage that VMS can safely be used to display Safety Campaign messages without compromising their use for incident management purposes.

\section{METHOD}

\section{University of Leeds Driving Simulator}

The study was performed using the new University of Leeds Driving Simulator (Figure 1). The simulator's vehicle cab is based around a 2005 Jaguar S-type, with all of its driver controls fully operational. A Seeing Machines faceLAB v4 eye-tracker is integrated within the cab, which itself is housed within a $4 \mathrm{~m}$ diameter, composite, spherical projection dome. A real-time, fully textured 3-D graphical scene of the virtual world is projected on the inner surface of the dome. The projection system that displays the visual information consists of eight channels, five forward and three rear views. The forward channels are edge-blended to provide a near seamless total horizontal field of view of $250^{\circ}$. The vertical field of view is $45^{\circ}$. The main rear channel $\left(40^{\circ}\right)$ is viewed through the vehicle's rear view mirror, whilst LCD panels are built in the Jaguar's wing mirrors to provide the two additional rear views.

The simulator incorporates an eight degree of freedom motion system. Lateral accelerations are simulated by sliding the whole vehicle cab and dome configuration along a railed gantry. Similarly, the whole gantry slides along tracks to create longitudinal acceleration cues. The $10 \mathrm{~m}-$ long rails and tracks allow $5 \mathrm{~m}$ of effective travel in each direction. In addition, sustained cues are provided by a standard $2.5 \mathrm{t}$ payload, electrically-driven hexapod. 


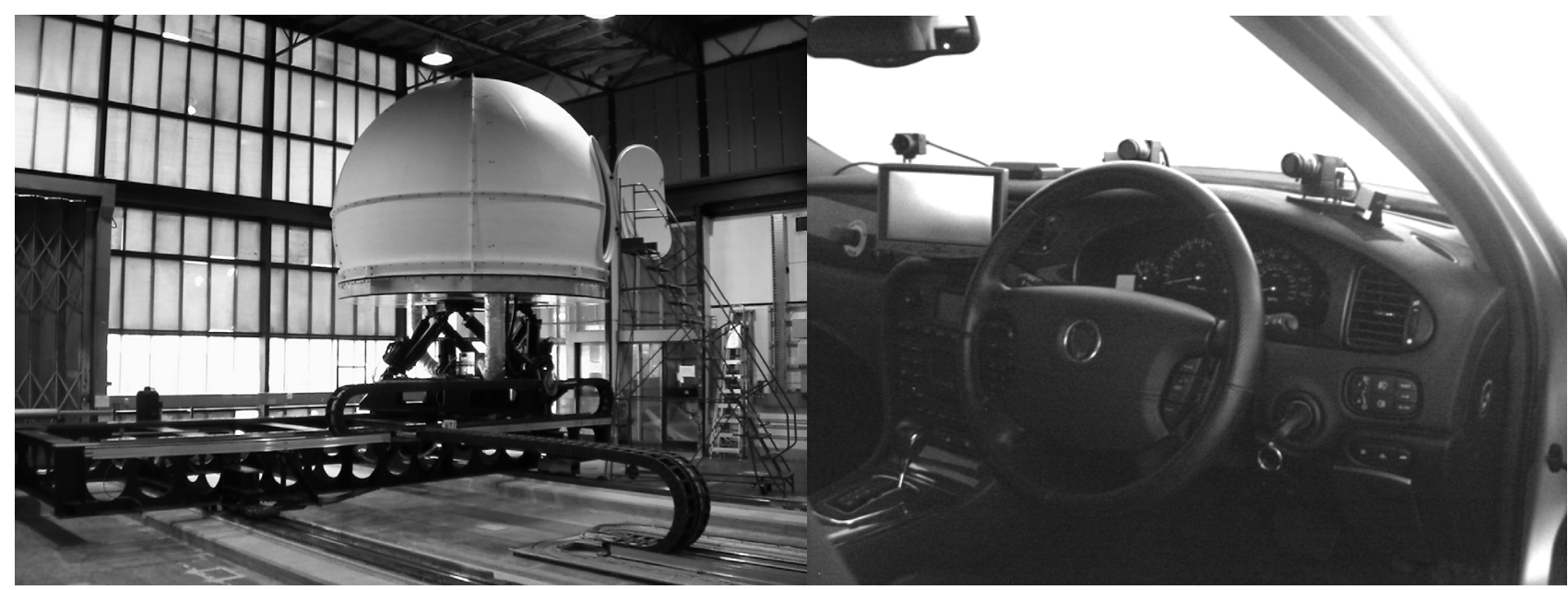

Figure 1. Exterior view and vehicle cab of the University of Leeds Driving Simulator

\section{Road network}

The virtual driving scenario consisted of a typical U.K. motorway with lane widths, markings and junction layouts as described in Chapter 5 (Road Markings) of the Traffic Signs Manual (2003). The virtual motorway consisted of both straight and curved segments (curve radius between $750 \mathrm{~m}$ and $1000 \mathrm{~m}$ ). The various segments made up four $9 \mathrm{~km}$ sections separated by four intersections. In each section, the VMS spacing was $1.5 \mathrm{~km}$, i.e., drivers passed six VMS per section and 24 throughout the whole drive.

A single experimental factor of Message Occurrence was defined as the proportion of active VMS within the total VMS witnessed. In the baseline condition ( $0 \%$ Message Occurrence), all VMS in that section were inactive (no message set), for 33\% Message Occurrence two per section were active, for $66 \%$ four per section and for $100 \%$ all six VMS were active. With four sections making up the total drive (24 VMS in total), drivers witnessed eight active VMS in the $33 \%$ Message Occurrence condition, sixteen active VMS in the $66 \%$ condition and all twentyfour in the $100 \%$ condition. Two Safety Campaign Messages were employed: "WATCH YOUR SPEED" and "KEEP YOUR DISTANCE." Equal numbers of each message were displayed throughout the road network.

After the last intersection, and when all the 24 active and inactive Safety Message VMS had been passed, a $25^{\text {th }}$ and final VMS displayed a Tactical Incident Management (TIM) message. When the driver passed a point $800 \mathrm{~m}$ before this final VMS, the TIM was activated. At this point, the particular lane in which the simulator driver was travelling was recorded. If the driver was travelling in either lane 1 or lane 2, the TIM displayed "ACCIDENT AHEAD - USE LANE 3." Similarly, if lane 3 was used, the TIM read "ACCIDENT AHEAD - USE LANE 1." This ensured that the simulator driver was forced to make a lane change on the advice of the VMS. Drivers were informed during the pre-experiment briefing that the left-most lane was lane 1, the middle was lane 2 and the right-most lane was lane 3 .

Surrounding traffic was present in adjacent lanes and each had a speed and headway that it preferred to maintain. The mean desired speed of ambient vehicles was $65 \mathrm{mph}$ in lane $1,75 \mathrm{mph}$ 


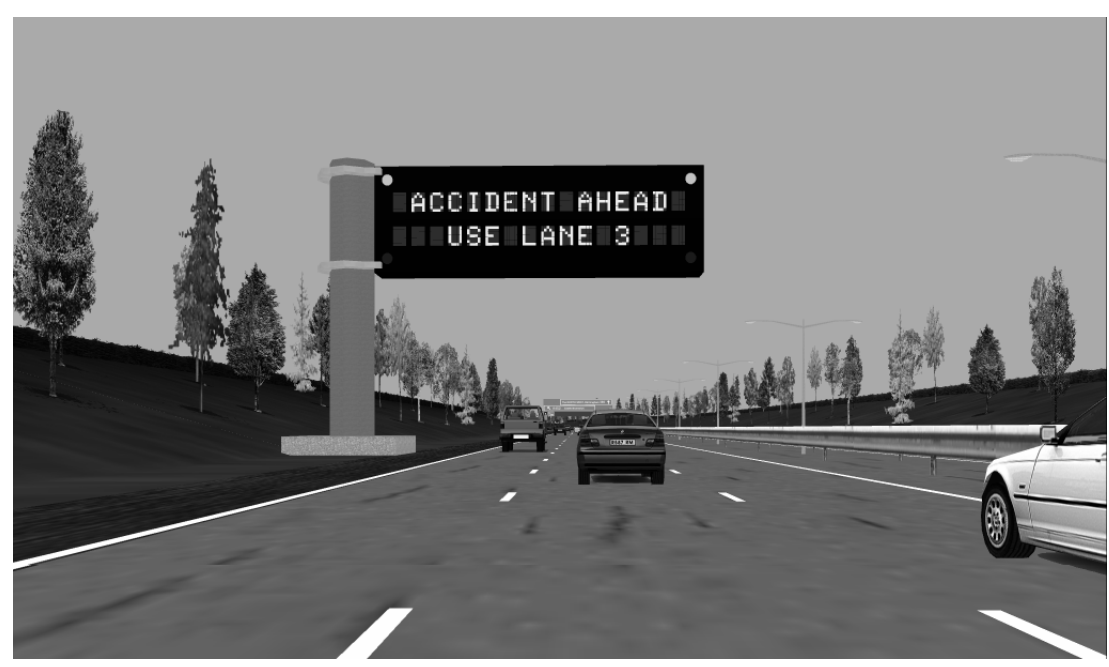

in lane 2 and $80 \mathrm{mph}$ in lane 3. All had a preferred mean headway of 3s. A normal distribution altered each individual vehicle's desired speed and headway from the mean. The appearance to the simulator driver was of realistic and well-behaved traffic with a lane count of around 1200 vehicles per hour per lane.

Figure 2. Visual scene including Tactical Incident Message

\section{Experimental procedure}

Eighty-two drivers were invited to take part in the study. They were selected on the proviso that they had held a driving licence for at least three years and drove in excess of 3,000 miles per year. None had driven the simulator prior to the study. Two participants withdrew during the practice period with symptoms of simulator sickness, giving a final sample of 80 drivers, distributed evenly into the four groups. Each group contained equal numbers of male and female drivers; groups were also split into 10 younger (35 years and less) and 10 older (over 35 ) drivers.

Participants drove the simulator twice during their visit. Firstly, they underwent a 15-minute practice session resembling the experimental motorway conditions. VMS were present but not active during the practice road. Next, each participant was allocated randomly to one of the four experimental groups of Message Concentration and undertook the $45 \mathrm{~km}$ drive under those conditions. At the end of the drive, following the TIM, participants were debriefed and paid $£ 10$ for their participation.

\section{Dependent variables}

Three areas of data collection surrounded each VMS. The legibility distance described the $250 \mathrm{~m}$ area prior to each sign over which it was possible for the simulator driver to read any message displayed on it. Upstream was defined as the $500 \mathrm{~m}$ of road preceding the start of the legibility distance and downstream the 500m following the end of the legibility distance.

The effectiveness of individual VMS displaying the two Safety Messages was investigated using speed change and headway change as the dependent variables. Speed change was defined as the difference between mean speed recording during the upstream and downstream regions.

Similarly, headway change was defined as the difference between mean following headway recording during the upstream and downstream regions. Under these definitions, an effective "Watch Your Speed" would produce a negative speed change, whilst an effective "Keep Your Distance" would produce a positive headway change. 
Driver response to the TIM was defined as time taken from this same start of legibility distance to when the vehicle first initiated lane change into the adjacent lane. The centre of gravity of the vehicle had to straddle a lane boundary for the lane change to be registered. A longer time implied reduced effectiveness of the TIM.

Eye-tracking provided the only physiological measure of driver performance. Data from both the simulator and the faceLAB v4 eye-tracker were post-processed to estimate the duration that a participant actually fixated on the VMS during the legibility distance. Gaze duration was only recorded whilst the vehicle was within the VMS legibility distance region. A longer time signified increased driver attention towards the sign.

\section{RESULTS}

\section{Safety Campaign Message "Watch Your Speed" (WYS)}

Table 1 shows the mean speed change (upstream - downstream) as participants experienced each sign activated and displaying the WYS Safety Campaign message in comparison to inactive blank signs. WYS1 refers to the first message that drivers witnessed, WYS2 the second, etc.

Table 1. Mean speed change (upstream - downstream) at each WYS presentation

\begin{tabular}{|l|c|c|c|c|c|c|c|c|c|c|c|c|}
\hline \multicolumn{1}{|c|}{ WYS } & $\mathbf{1}$ & $\mathbf{2}$ & $\mathbf{3}$ & $\mathbf{4}$ & $\mathbf{5}$ & $\mathbf{6}$ & $\mathbf{7}$ & $\mathbf{8}$ & $\mathbf{9}$ & $\mathbf{1 0}$ & $\mathbf{1 1}$ & $\mathbf{1 2}$ \\
\hline Inactive & 0.681 & -0.216 & 0.73 & 0.605 & -0.499 & 0.745 & 0.58 & 1.07 & -0.222 & 0.832 & -0.127 & 0.494 \\
\hline Active & -0.577 & -0.396 & -0.844 & 0.288 & 0.239 & 0.264 & -0.512 & 0.132 & 0.273 & -0.153 & 0.251 & -0.667 \\
\hline
\end{tabular}

T-tests were performed at each of the twelve locations to investigate whether there was a significant difference in speed change dependent on sign activity. In all but three of the twelve cases at an individual presentation (WYS5, WYS9 and WYS11), the effect of the Safety Message was to reduce driving speed compared to an inactive, blank VMS. However, this speed reducing effect instigated by sign activity was never significant beyond the $95 \%$ confidence level, only approaching such a level at WYS3 (93.5\% confidence). Even if the significance at this one presentation had been more frequently observed, the impact of the message remains limited: the difference between the inactive and active speed reductions was a mere $0.5 \mathrm{mph}$.

\section{Safety Campaign Message "Keep Your Distance" (KYD)}

The main dependent variable used was the change in mean following headway downstream compared to upstream of the VMS location. Results are shown in Table 2. T-tests were also performed at each sign location to investigate whether there was a significant change in headway if a sign was active with a KYD message or not.

Table 2. Mean headway change (upstream - downstream) at each KYD presentation

\begin{tabular}{|l|c|c|c|c|c|c|c|c|c|c|c|c|}
\hline KYD & $\mathbf{1}$ & $\mathbf{2}$ & $\mathbf{3}$ & $\mathbf{4}$ & $\mathbf{5}$ & $\mathbf{6}$ & $\mathbf{7}$ & $\mathbf{8}$ & $\mathbf{9}$ & $\mathbf{1 0}$ & $\mathbf{1 1}$ & $\mathbf{1 2}$ \\
\hline Inactive & -0.13 & 0.0051 & -0.21 & -0.039 & 0.013 & 0.045 & -0.089 & -0.114 & -0.071 & -0.009 & -0.051 & 0.0016 \\
\hline Active & 0.055 & -0.079 & 0.061 & 0.064 & 0.0091 & -0.093 & 0.060 & -0.011 & 0.021 & 0.011 & -0.064 & -0.11 \\
\hline
\end{tabular}


In most cases at a particular presentation, the effect of the Safety Message was to increase headway greater than an inactive sign. As for WYS, this was particularly true for the early presentations of the message, although the magnitude of their effect was again small (on average around $0.05 \mathrm{~s}$ improvement between active and inactive signs).

\section{Effect of Message Occurrence on response to a Tactical Incident Message}

The effect of Message Concentration on driver response to the TIM was evaluated using the time

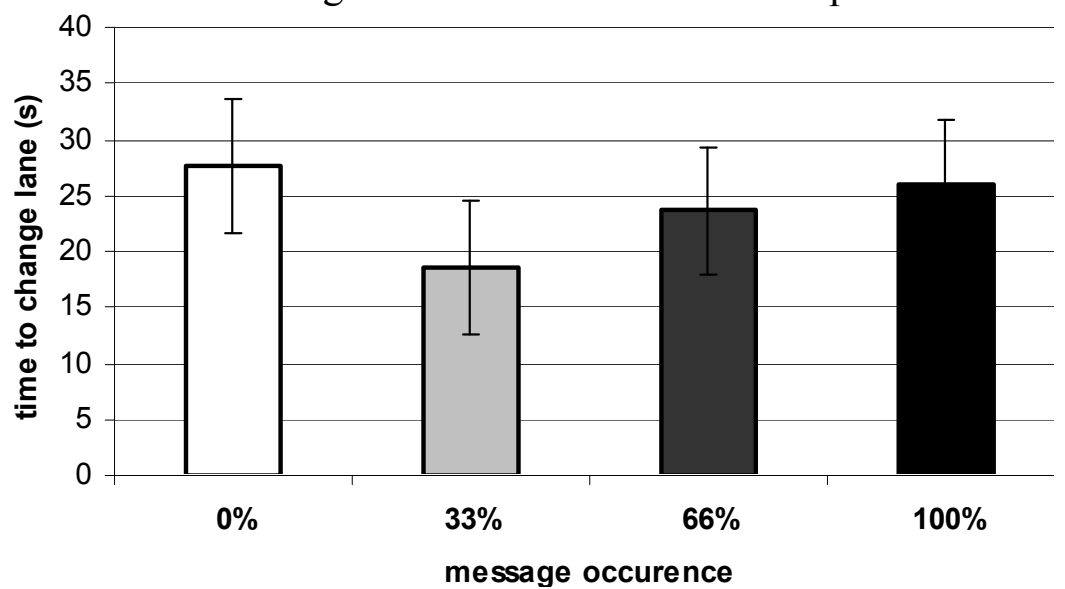
taken to make an effective lane change. Results shown in Figure 3 demonstrate a significant main effect, $\mathrm{F}(3,79)=3.39, \mathrm{p}=.034$. In the $33 \%$ condition, the required lane change was made significantly quicker than when the driver had experienced all previous VMS that were blank $(0 \%)$ or all carrying Safety Campaign messages (100\%).

Figure 3. Response time to change lane on the advice of the TIM

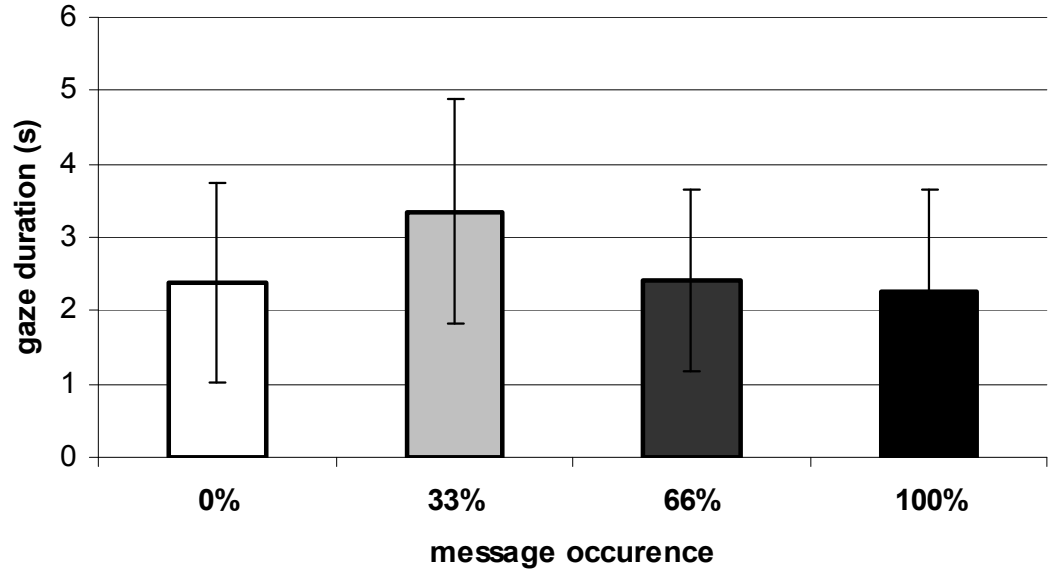

Figure 4. Gaze duration with message occurrence
Figure 4 shows the gaze duration throughout the legibility distance for the TIM across each of the four conditions of Message Occurrence. Post-hoc tests showed that drivers in the $33 \%$ group gazed at the TIM for a significantly longer duration than the $0 \%$ group $(\mathrm{p}=.053)$, the $66 \%$ group $(\mathrm{p}=.027)$ or the $100 \%$ group $(\mathrm{p}=0.047)$.

\section{DISCUSSION}

The first aim of the study was to investigate the immediate impact of Safety Campaign messages when displayed on VMS. At an individual level, results from the study did not suggest that either of the messages "Watch Your Speed" (WYS) or "Keep Your Distance" (KYD) were particularly effective in altering driver behaviour. WYS did have the potential to reduce speed by a maximum of around $0.5 \mathrm{mph}$ and KYD to increase headway by around $0.05 \mathrm{~s}$, but even these limited effects were associated with the initial presentations of the messages, whilst they still retained some novelty value to drivers. Even with the fairly substantial dataset of eighty drivers 
used in this study, the statistical significance of the results, even when considering the signs in combination, rarely proved to be greater than the $95 \%$ confidence level. Overall, this would suggest that Safety Campaign messages at an individual level had little influence over the driving population. The fact that results from the three on-road trials mirrored these findings adds confidence to this conclusion.

The presentation of the Safety Campaign messages did, however, appear to have a positive impact on driver alertness. The eye-tracking data recorded in the study showed that drivers continued to fixate on VMS carrying repeated safety messages, even though their presentation did not particularly influence their driving performance. This level of alertness allowed drivers who had witnessed a smattering of active VMS earlier in their journey to respond more appropriately to a Tactical Incident Message (TIM) than those who had not witnessed any active VMS.

Regarding the third and final aim of the investigation, it was demonstrated than it is possible for drivers to become desensitised through over-use of the safety messages. Drivers who experienced either no safety messages or those that witnessed many both tended to ignore or respond slower to a TIM. In both of these cases, this inferior behaviour correlated with shorter gaze durations towards the message, which may well have been due to a late recognition of the text. The drivers who had experienced no signs were probably a little 'out-of-the-loop' and hence took longer to respond, whereas those who had endlessly seen the same message may well have, consciously or not, diverted attention away from signs.

In summary, it would appear that VMS carrying Safety Campaign Messages do not significantly alter driver behaviour, in that drivers do not actively change their driving style on the advice of the sign. That said, they do still appear to carry a safety benefit. When used sporadically, they can improve driver alertness and act as an opportune reminder of a safe driving practice. The evidence in this study also suggests that a driver's response to Tactical Incident Messages can be more timely having becoming accustomed to reading such messages on VMS but without having become jaded with their content.

\section{REFERENCES}

Garber, N.J. and Patel, S.T. (1995). Control of vehicle speeds in temporary traffic control zones (work zones) using changeable message signs with radar. Transportation Research Record, 1509, 73-81.

Highways Agency. (2005a). Effectiveness of VMS Safety Messages - Trial in October 2004. Report prepared by Mott MacDonald, Capital House, Winchester, U.K. January 2005.

Highways Agency. (2005b). Effectiveness of VMS Safety Messages - On Road Trial June/July 2005. Report prepared by Mott MacDonald, Capital House, Winchester, U.K. November 2005.

Highways Agency. (2006). Effectiveness of VMS Safety Messages - On Road Trial January/February 2006. Report prepared by Mott MacDonald, Winchester, U.K. March 2006.

Rama, P. and Kulmala, R. (2000). Effects of variable message signs for slippery road conditions on driving speed and headways. Transportation Research Part F, 3, 85-94. 